\title{
Effect of berberine on the renal tubular epithelial- to-mesenchymal transition by inhibition of the Notch/snail pathway in diabetic nephropathy model KKAy mice
}

\author{
This article was published in the following Dove Press journal: \\ Drug Design, Development and Therapy \\ 31 March 2017 \\ Number of times this article has been viewed
}

\section{Guannan Yang \\ Zongjiang Zhao \\ Xinxue Zhang \\ Amin Wu \\ Yawei Huang \\ Yonghui Miao \\ Meijuan Yang}

School of Basic Medical Science,

Beijing University of Chinese

Medicine, Beijing, People's

Republic of China
Correspondence: Zongjiang Zhao

School of Basic Medical Science, Beijing

University of Chinese Medicine, II Bei

San Huan Dong Lu, Chaoyang, 100029

Beijing, People's Republic of China

Tel +86 I39 I050 7288

Email zongjiangz@sina.com

\begin{abstract}
Renal tubular epithelial-to-mesenchymal transition (EMT) and renal tubular interstitial fibrosis are the main pathological changes of diabetic nephropathy (DN), which is a common cause of end-stage renal disease. Previous studies have suggested that berberine (BBR) has antifibrotic effects in the kidney and can reduce apoptosis and inhibit the EMT of podocytes in DN. However, the effect of BBR on the renal tubular EMT in DN and its mechanisms of action are unknown. This study was performed to explore the effects of BBR on the renal tubular EMT and the molecular mechanisms of BBR in DN model KKAy mice and on the high glucose (HG)-induced EMT in mouse renal tubular epithelial cells. Our results showed that, relative to the model mice, the mice in the treatment group had an improved general state and reduced blood glucose and 24-h urinary protein levels. Degradation of renal function was ameliorated by BBR. We also observed the protective effects of BBR on renal structural changes, including normalization of an index of renal interstitial fibrosis and kidney weight/body weight. Moreover, BBR suppressed the activation of the Notch/snail pathway and upregulated the $\alpha$-SMA and E-cadherin levels in DN model KKAy mice. BBR was further found to prevent HG-induced EMT events and to inhibit the HG-induced expression of Notch pathway members and snaill in mouse renal tubular epithelial cells. Our findings indicate that BBR has a therapeutic effect on DN, including its inhibition of the renal tubular EMT and renal interstitial fibrosis. Furthermore, the BBR-mediated EMT inhibition occurs through Notch/snail pathway regulation.
\end{abstract}

Keywords: berberine, EMT, renal interstitial fibrosis, diabetic nephropathy, Notch/snail pathway

\section{Introduction}

Diabetes mellitus (DM) has become the third most common chronic noncommunicable disease threatening human health, behind cardiac/cerebrovascular disease and malignant tumors. ${ }^{1}$ Diabetic nephropathy (DN) is one of the most important microvascular complications of DM. Globally, $40 \%$ of DN patients ultimately progress to end-stage renal disease and half of the end-stage renal disease patients exhibit $\mathrm{DN}$. $^{2,3}$

Renal interstitial fibrosis is the final process of DN, the main feature of which is atrophy of renal tubules. Renal tubular epithelial-to-mesenchymal transition (EMT) is a unique event in the process of renal fibrosis and a relatively complex pathological process. To combat damage and avoid potential apoptosis, some renal tubular epithelial cells lose their epithelial cell markers, change their morphology, become muscle fibroblasts, and produce the mesenchymal cell marker alpha-smooth muscle actin 
( $\alpha$-SMA). This pathologic process, which involves a series of signaling pathways and multiple cytokines, is complex and dynamic. Studies of these pathways are necessary to explore and identify the related factors and to perform appropriate intervention measures against DN.,5

The Notch pathway has been confirmed to mediate epithelial cell EMT and other cellular fibrosis in DN, and has been associated with transforming growth factor (TGF)- $\beta 1 .^{6}$ In addition, Murea et $\mathrm{al}^{7}$ found that activation of the Notch pathway is a common mechanism of proteinuria in renal disease and is widely present in the pathophysiology of glomerular sclerosis and renal tubular interstitial fibrosis. Additionally, based on a large number of basic and clinical trials, the Notch pathway plays an important role in the process of EMT. ${ }^{8,9}$ Evidence suggests that the Notch pathway induces the EMT independently in DN. ${ }^{10}$ Relative to the control group, the expression of jagged 1 and hes 1 in patients with DN is significantly increased. ${ }^{6}$ Researchers have suggested that the notch1 and TGF- $\beta_{1}$ pathways may be the key to the development of DN. ${ }^{10}$ Several cell system studies have revealed that snail directly inhibits the transcription of E-cadherin (E-Cad) and downregulates its expression, which causes the epithelial cells to lose their adhesion and promotes the occurrence of EMT. It is believed that snail plays an important role in regulating the gene expression during the process of cell differentiation and directly leads to the generation of mesenchymal-like cells. ${ }^{11-14}$ Snail is regulated by many signaling pathways, the most important one being the Notch signal pathway. Recent research also shows that the Notch pathway induces EMT by activating snail. The expression of snail 1 is regulated directly by the Notch signaling pathway. ${ }^{15}$ The Notch/snail pathway is an important mechanism in the process of DN renal interstitial fibrosis and is an important target for the drug regulation and control of interstitial fibrosis.

Modern medicine for DN treatment is mainly used to control the blood glucose and blood pressure and to regulate the lipid metabolism. Drugs are mostly used to treat early DN. ${ }^{16-20}$ Berberine (BBR) is an isoquinoline alkaloid isolated from Coptidis rhizome and Cortex phellodendri. ${ }^{21,22}$ In traditional Chinese medicine (TCM), BBR has been commonly used as an oral drug to treat gastroenteritis and secretory diarrhea for more than 1,400 years. In recent years, BBR has been widely used to treat diabetes and its complications. ${ }^{23,24}$ BBR treatment may restore the renal functional parameters, improve the glucose and lipid metabolism disorders, and suppress alterations of histologic and ultrastructural changes in the kidney. ${ }^{25}$ Moreover, BBR inhibits mesangial cell proliferation and extracellular matrix accumulation induced by high glucose (HG) and ameliorates tubulointerstitial fibrosis in DN, which suggests that BBR can be used as a potential drug for DN. ${ }^{26-30}$ Most importantly, a recent study has shown that BBR protects human renal proximal tubular cells from hypoxia/reoxygenation injury. ${ }^{31}$

Thus, the effect of BBR is extensive, with multiple targets. The mechanisms through which BBR inhibits EMT and renal interstitial fibrosis in renal tubular epithelial cells in DN remain unclear. Therefore, in this study, we investigated the protective effects of BBR against renal injury and explored the effects of BBR on tubular epithelial transdifferentiation by measuring changes in the protein and messenger RNA (mRNA) levels of jagged1, notch1, hes1, E-Cad, and $\alpha$-SMA in the kidney tissue of DN model KKAy mice following BBR administration. Further investigation of the molecular mechanisms underlying the efficacy of BBR as a clinical treatment for DN is recommended.

\section{Materials and methods Chemicals and reagents}

BBR hydrochloride tablets were purchased from the Chengdu Jinhua Pharmaceutical Co., Ltd. (Chengdu, People's Republic of China). Each tablet contained $0.1 \mathrm{~g}$ of BBR hydrochloride as the main ingredient, and the excipients were starch, colloidal silica, hydroxypropylmethylcellulose, polysorbate 80 , sodium carboxymethyl starch, magnesium stearate, and ethanol.

\section{Experimental animals and treatment}

All experiments were performed in accordance with the National Institutes of Health guidelines (NIH Pub. No. 85-23, revised 1996) concerning the care and treatment of experimental animals. The study was approved by the Animal Research Committee of the Beijing University of Chinese Medicine. Twenty female KKAy mice (8-10 weeks of age) weighing 25-28 g were used in the current experiments. Ten female C57BL/6J mice (8-10 weeks of age) weighing 23-25 g were used as age-matched controls. All mice were purchased from Beijing HFK Bioscience Co., Ltd. (Beijing, People's Republic of China) and were raised in the animal houses of the Beijing University of Chinese Medicine (Beijing, People's Republic of China). After all the mice were fed adaptively for 1 week, the KKAy mice were allowed access to KK feed (Beijing HFK Bioscience Co., Ltd.) and water ad libitum. To serve as a control, the $\mathrm{C} 57 \mathrm{BL} / 6 \mathrm{~J}$ mice were fed a normal diet and allowed ad libitum access to water until the end of the experiment. After 10 weeks of induction, blood samples were collected from the tail vein 
to measure the blood glucose and $24 \mathrm{~h}$ urine was collected from the mice in the metabolic cage. The criteria for identification as a suitable DN animal model were random blood glucose $>16.7 \mathrm{mmol} / \mathrm{L}$ and $24 \mathrm{~h}$ urinary protein $>0.4 \mathrm{mg}$. The DN model KKAy mice were randomly divided into model group ( $\mathrm{MG}, \mathrm{n}=10)$ and treatment group ( $\mathrm{TG}, \mathrm{n}=10)$, with an equivalent distribution of average body weights and blood glucose levels between the two groups. The C57BL/6J mice were used as the normal control group $(\mathrm{NG}, \mathrm{n}=10)$. The TG was given BBR $(150 \mathrm{mg} / \mathrm{kg} / \mathrm{d})$ by gavage administration, while the $\mathrm{MG}$ received an equivalent volume of saline for 16 weeks. The mice were housed individually in plastic cages in a clean animal cabinet with ad libitum access to food and water throughout the experimental periods. Body weight measurements were conducted every 4 weeks. Blood samples for the determination of blood glucose levels were taken from the tip of the tail in the 26th week using the Roche blood glucose meter and test strips (Hoffmann-La Roche Ltd., Basel, Switzerland). In the 26th week, all the mice were anesthetized with an intraperitoneal injection of $4 \%$ hydrazine acid $(0.1 \mathrm{~mL} / 10 \mathrm{~g})$. Blood was retro-orbitally collected, stored for $2 \mathrm{~h}$ at $4^{\circ} \mathrm{C}$, and centrifuged (2,500 rpm) for $20 \mathrm{~min}$ at $4^{\circ} \mathrm{C}$; the upper serum was collected and stored at $-20^{\circ} \mathrm{C}$ until analysis. The mouse kidney was sterilely removed, followed by sector slitting, and the kidney tissues were separately fixed in 10\% neutral formalin and special $10 \%$ neutral formalin for in situ hybridization (ISH). The remaining tissues were quickly frozen in liquid nitrogen and then preserved at $-80^{\circ} \mathrm{C}$ for Western blotting assays.

\section{Cell culture}

Mouse renal tubular epithelial cells (mRTECs) were purchased from the American Type Culture Collection (Manassas, VA, USA). The cells were maintained in Dulbecco's Modified Eagle's medium (DMEM) (low glucose; Gibco Life Technologies, Grand Island, NY, USA) with $10 \%$ FBS (HyClone) in a humidified atmosphere containing $5 \% \mathrm{CO}_{2}$ at $37^{\circ} \mathrm{C}$. After growing to $\sim 80 \%$ confluence, the mRTECs were cultured in serum-free DMEM for $24 \mathrm{~h}$ at $37^{\circ} \mathrm{C}$ to arrest and synchronize cell growth. In vitro, the cells were divided into the following four groups: 1) $\mathrm{NG}$, in which the cells were treated with DMEM containing $5.5 \mathrm{mM} / \mathrm{L}$ glucose; 2) HG group, in which the cells were treated with $30 \mathrm{mM} / \mathrm{L}$ glucose; 3) BBR group, in which the cells were treated with $30 \mathrm{mM} / \mathrm{L}$ glucose and $30 \mu \mathrm{MBBR} ;{ }^{26}$ and 4) DAPT group, in which the cells were treated with $30 \mathrm{mM} / \mathrm{L}$ glucose after pretreatment with $10 \mu \mathrm{M} / \mathrm{L} N-[N-(3,5-$ difluorophenacetyl)-L-alanyl]-S-phenylglycine $t$-butyl ester
(DAPT; Sigma-Aldrich, Darmstadt, Germany) for $40 \mathrm{~min}$. The mRTECs of the four groups were treated for $48 \mathrm{~h}$ and then used for the purposed experiments.

\section{Metabolic and biochemical parameters}

At 26 weeks of age, all the mice were deprived of food pellets for $10 \mathrm{~h}$ and blood was retro-orbitally collected. Blood urea nitrogen (BUN) and plasma creatinine $(\mathrm{Cr})$ levels were measured using an automated biochemical analyzer (Hitachi Ltd., Tokyo, Japan).

\section{Urine albumin analysis}

Mice were transferred to metabolic cages for $24 \mathrm{~h}$ to collect the urine samples every 4 weeks using the Liquid Protein Extraction Kit-II (P1255; Applygen Technologies, Inc., Beijing, People's Republic of China) to extract urine, excluding the interference of uric acid and other impurities. Urine albumin concentrations were determined using the bicinchoninic acid protein quantification assay kit (P1511, Applygen Technologies, Inc.). Albuminuria in the mice was expressed as milligrams per $24 \mathrm{~h}$.

\section{Kidney histology}

Kidney sections were fixed in 10\% neutral buffered formalin, embedded in paraffin, and cut into $4 \mu \mathrm{m}$ thick sections, which were prepared for hematoxylin and eosin (HE) and Mallory staining. HE staining: Paraffin sections were conventionally dewaxed in conventional dewaxing water, immersed in hematoxylin for $1 \mathrm{~min}$, subjected to liquid differentiation for $5 \mathrm{~s}$, and immersed in eosin for $2 \mathrm{~min}$, followed by neutral gum sealing. Mallory staining: paraffin sections were conventionally dewaxed in dewaxing water, immersed in Mordant dyeing liquid for $1 \mathrm{~h}$ at room temperature, immersed in Mallory I dye for $2 \mathrm{~min}$, and immersed in Mallory II dye for $20 \mathrm{~min}$, followed by neutral gum sealing.

\section{Electron microscopy}

Three specimens in each group were cut into small pieces $(1 \times 1 \times 1 \mathrm{~mm})$. These pieces were washed with sodium cacodylate buffer, fixed in $2.5 \%$ glutaraldehyde, dehydrated with a graded series of ethanol, sectioned with an ultramicrotome (Leica, Witzla, Germany), and stained with saturated uranium acetate. Transmission electron microscopy (TEM) was performed using a JEM 100CX electron microscope (JEOL, Tokyo, Japan).

\section{Immunohistochemistry}

Kidney tissues were fixed in 10\% neutral formalin, embedded in paraffin, sliced $(3 \mu \mathrm{m})$, dewaxed, washed three times with 
phosphate-buffered saline (PBS) for 5 min each, incubated in $0.01 \%$ Triton for $8 \mathrm{~min}$ and in $3 \%$ hydrogen dioxide solution for $10 \mathrm{~min}$, antigen repaired with citrate buffer solution in a microwave, blocked with $10 \%$ goat serum, and incubated with primary antibodies against jagged 1 (1:100 dilution; Novus, Littleton, CO, USA), notch1 (1:100 dilution; Abcam, Cambridge, UK), hes1 (1:125 dilution; CST, Danvers, MA, USA), snail1 (1:100 dilution, Novus), E-Cad (1:100 dilution, Abcam), and $\alpha$-SMA (1:200 dilution, Abcam) overnight at $4^{\circ} \mathrm{C}$. The sections were washed three times with PBS for $5 \mathrm{~min}$, after which they were incubated in goat anti-rabbit IgG (1:1,000 dilution, Abcam) or goat anti-mouse IgG bound to horseradish peroxidase (HRP; 1:1,000 dilution, Abcam) for $0.5 \mathrm{~h}$, washed three times with PBS, and stained with diaminobezidin (DAB) for 5-10 min. Images were obtained with a light microscope (Ni-U; Nikon Corporation, Tokyo, Japan). The images were analyzed quantitatively by Image-Pro Plus 6.0 (Media Cybernetics, Inc., Washington, DC, USA). For each group, 8-15 fields were randomly selected to determine the optical density value.

\section{In situ hybridization}

All the instruments used in the ISH test were treated with diethypyrocarbonate (DEPC), and all the reagents were prepared using DEPC. In situ expression analyses were performed using the RNAscope ISH kit from Wuhan Boster Biological Engineering Co., Ltd. (Wuhan, People's Republic of China). Kidney slices for ISH were stored at $-20^{\circ} \mathrm{C}$, rewarmed, dewaxed, washed three times with PBS for 5 min each, incubated with $0.01 \%$ Triton for $10 \mathrm{~min}$ and a $3 \%$ hydrogen dioxide solution for $10 \mathrm{~min}$, digested by pepsin freshly diluted with $3 \%$ citric acid for 6 min, washed three times with PBS, incubated with a prehybridization solution for $2 \mathrm{~h}$ and a hybridization solution for $5 \mathrm{~h}$ at $37^{\circ} \mathrm{C}$, washed three times with $2 \times \mathrm{SSC}, 1 \times \mathrm{SSC}$, and $0.2 \times$ saline sodium citrate (SSC) for 5 min successively, blocked with a special sealing liquid for $30 \mathrm{~min}$, and incubated with biotin-labeled digoxin overnight at $4^{\circ} \mathrm{C}$. Ultrasensitive peroxidase was used for incubation for $20 \mathrm{~min}$, after which the slices were incubated in HRP for 20 min, washed three times with PBS, and stained with DAB for $15 \mathrm{~min}$. The imaging and statistical analyses were the same as those used for immunohistochemistry.

\section{Western blot analysis}

Renal cortical tissues $(\sim 100 \mathrm{mg})$ and mRTECs were collected and lysed with radioimmunoprecipitation assay buffer, the lyses were clarified by centrifugation, and the supernatants were collected. Protein concentrations were determined using the bicinchoninic acid protein assay (Applygen
Technologies, Inc.). Equivalent amounts of protein $(50 \mu \mathrm{g})$ were resolved on sodium dodecyl sulfate polyacrylamide gels and transferred by electroblotting to polyvinylidene difluoride membranes. The membranes were blocked in 5\% (W/V) nonfat milk (Applygen Technologies, Inc.) at room temperature for $1 \mathrm{~h}$, after which they were incubated overnight at $4^{\circ} \mathrm{C}$ with specific primary antibodies against jagged 1 (1:1,000 dilution, Novus), notch1 (1:2,000 dilution, Abcam), hes1 (1:500 dilution, CST), snail1 (1:1,000 dilution, Novus), E-Cad (1:1,000 dilution, Abcam), and $\alpha$-SMA (1:2,000 dilution, Abcam). The membranes were washed in Tris-buffered saline (TBS)-T (Tween 20$)$ buffer (0.1\% TBS-T; TBS with $0.1 \%$ Tween 20 ) three times for $10 \mathrm{~min}$ each and in TBS one time for $10 \mathrm{~min}$, and incubated with HRP-linked anti-mouse secondary antibodies (1:6,000 dilution, Abcam) or anti-rabbit secondary antibodies (1:3,000 dilution, Abcam). The membranes were washed in $0.1 \%$ TBS-T and TBS, after which the immunolabeled proteins were detected with electrochemiluminescence (ECL) solution (EMD Millipore, Billerica, MA, USA). The density of the detected bands was analyzed using ImageJ software (National Institutes of Health, Bethesda, MD, USA).

\section{Real-time polymerase chain reaction}

Total mRTEC RNA was isolated using TRIzol reagent (Invitrogen) according to the manufacturer's instructions. Then, the RNA was reverse-transcribed into complementary DNA using the Reverse Transcription System (Promega Corporation, Madison, WI, USA). Relative mRNA levels were examined using SYBR Green realtime quantitative reverse transcription-polymerase chain reaction (qRT-PCR; Bio-Rad, Hercules, CA, USA) and were calculated by the $2^{-\Delta \Delta \mathrm{Ct}}$ method. The primer sequences are as follows: mouse E-Cad: forward primer, 5'-GGTTTTCTACAGCATCACCG-3' and reverse primer, 5'-CACAGTAGTTTACCCCTTCG-3'; mouse $\alpha$-SMA: forward primer, 5'-TGTGCTGGACTCTGGAGATG-3' and reverse primer, 5'-GATCACCTGCCCATCAGG-3'; mouse jagged1: forward primer, 5'-CCTCGGGTCAGTTT GAGCTG-3' and reverse primer, 5'-CCTTGAGGCACA CTTTGAAGTA-3'; mouse notch1: forward primer, 5'-GAGGCGTGGCAGACTATCATGC-3' and reverse primer, 5'-CTTGTACTCCGTCAGCGTGA-3'; mouse hes1: forward primer, 5'-CCAGCCAGTGTCAACACGA-3' and reverse primer, 5'-AATGCCGGGAGCTATCTTTCT-3'; mouse snail1: forward primer, 5'-CACACGCTGCCTTGT GTCT-3' and reverse primer, 5'-GGTCAGCAAAAGCAC GGTT-3'; and mouse $\beta$-actin: forward primer, 5'-TCCT GTGGCATCCACGAAACT-3' and reverse primer, 5'-GAAGCATTTGCGGTGGACGAT-3'. All RT-PCRs 
Table I Effect of berberine on the body weight of diabetic nephropathy model KKAy mice (g)

\begin{tabular}{|c|c|c|c|c|c|}
\hline \multirow[t]{2}{*}{ Group } & \multicolumn{5}{|c|}{ Body weight (g) } \\
\hline & I0th week & 14th week & I8th week & 22nd week & 26th week \\
\hline NG & $21.72 \pm 0.81$ & $24.04 \pm 1.33$ & $24.40 \pm .80$ & $24.36 \pm 1.07$ & $25.27 \pm 1.29$ \\
\hline MG & $43.77 \pm 7.18^{* *}$ & $52.83 \pm 4.05^{* *}$ & $56.87 \pm 4.55^{* *}$ & $55.50 \pm 5.12^{* *}$ & $55.60 \pm 3.73 * *$ \\
\hline TG & $21.72 \pm 4.50 * *$ & $50.85 \pm 7.18^{* *}$ & $53.58 \pm 2.43$ **,\# & $53.50 \pm 1.87^{* *}$ & $52.50 \pm 2.70 * *$, \# \\
\hline
\end{tabular}

Notes: Data are presented as mean \pm standard deviation. ${ }^{* * P} P<0.01$ compared with the NG and ${ }^{\# P} P 0.01$, ${ }^{\#}<<0.05$ compared with the MG.

Abbreviations: MG, model group; NG, normal control group; TG, treatment group.

were performed in triplicate, and the data are presented as the mean \pm standard deviation (SD).

\section{Statistical analysis}

The data are presented as the mean \pm SD. Statistical analyses were performed using SPSS 20.0. The differences among multiple groups were evaluated by one-way analyses of variance. $P<0.05$ was considered statistically significant.

\section{Results}

\section{Effect of BBR on the general state of DN model KKAy mice}

Continuously fed KK feed, DN model KKAy mice appeared listless, exhibited reduced activity, displayed a lackluster coat, drank more, consumed more food, and had a greater urinary output. At $\sim 10$ weeks, diabetic complications, such as skin ulcers and urinary tract infections, appeared. Relative to the MG, the general state of the TG was improved. In this experiment, the mice were weighed once every 4 weeks. As shown in Table 1 and Figure 1, as the experiment was conducted, the body weight of the DN model KKAy mice gradually increased, and a significant difference in body weight emerged between the DN mice and the C57BL/6J mice $(P<0.01)$. Although the weight of the mice in the TG also increased slowly, after 16 weeks of treatment, the weight of the TG was significantly reduced relative to the $\mathrm{MG}(P<0.01)$.

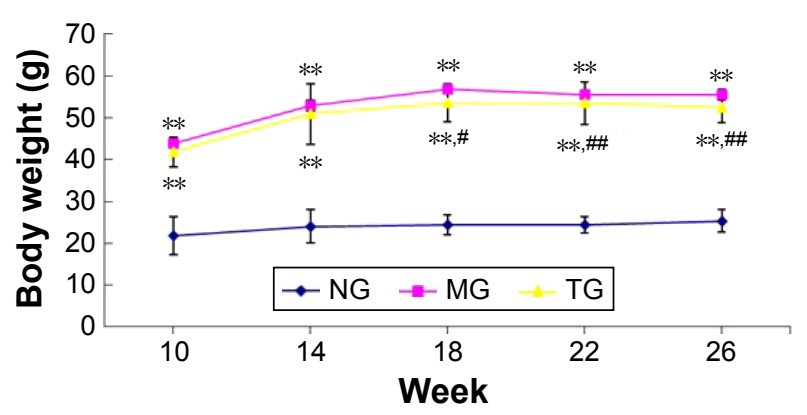

Figure I Effect of berberine on the body weight of DN model KKAy DN mice. Notes: Data are presented as mean \pm standard deviation. $* * P<0.01$ compared with the NG and ${ }^{\#} P<0.01,{ }^{\#} P<0.05$ compared with the MG.

Abbreviations: DN, diabetic nephropathy; MG, model group; NG, normal control group; TG, treatment group.
Effects of BBR on the metabolic and biochemical parameters in DN model KKAy mice

The kidney weight/body weight ratio $(\mathrm{KW} / \mathrm{BW})$ in the DN model KKAy mice was significantly higher than in the TG (Table 2; $P<0.01$ ), and there was a decrease in the random blood glucose in the group treated with BBR $(P<0.05)$. At the end of the 26th week, the blood serum $\mathrm{Cr}$ and $\mathrm{BUN}$ were increased in the DN model KKAy mice (Table 2). After 16 weeks of therapy, the levels of $\mathrm{Cr}$ and BUN were dramatically reduced in the BBR-treated mice relative to the DN mice (Table 2; $P<0.01$ ). All the above results indicated that $\mathrm{BBR}$ has a protective effect on the kidney function in DN.

\section{Effects of BBR on albuminuria in DN model KKAy mice}

Similar to the body weight, $24 \mathrm{~h}$ urine protein was detected every 4 weeks (Table 3; Figure 2). As time progressed, the $24 \mathrm{~h}$ urine protein of the MG increased gradually, and it was significantly higher than that of the $\mathrm{C} 57 \mathrm{BL} / 6 \mathrm{~J}$ mice in the control group at 14 weeks $(P<0.01)$. Relative to the MG, the $24 \mathrm{~h}$ urinary protein in the BBR group was significantly decreased $(P<0.01)$. Although there was an increase in urinary protein content during the 22nd and the 26th weeks, the mean urinary protein content in the TG of mice was significantly lower than that in the MG, as evidenced by measurements taken at 18,22, and 26 weeks $(P<0.01)$, and the growth rate was significantly decreased relative to the MG. The above data clearly indicate that BBR can delay the progression of renal dysfunction and protect the renal function.

\section{Effect of BBR on the morphologic changes in the kidneys of DN model KKAy mice}

Kidney tissues were collected for HE and Mallory staining at the end of the experiment. HE staining revealed normal glomerular and tubular structures in the normal group (Figure 3A). In the MG, a variety of DN-induced changes in renal morphology were detected (Figure 3B), such as glomerular hypertrophy, mesangial cell proliferation, 
Table 2 Effect of berberine on the metabolic and biochemical parameters in diabetic nephropathy model KKAy mice

\begin{tabular}{lllll}
\hline Group & RBG $(\mathbf{m m o l} / \mathbf{L})$ & KW/BW $(\mathbf{m g} / \mathbf{g})$ & BUN $(\mathbf{m m o l} / \mathbf{L})$ & Serum Cr $(\mu \mathrm{mol} / \mathbf{L})$ \\
\hline NG & $4.52 \pm 0.46$ & $2.74 \pm 0.28$ & $48.84 \pm 1.03$ & $5.86 \pm 5.24$ \\
MG & $21.65 \pm 7.23^{* *}$ & $6.91 \pm 0.14^{* *}$ & $69.05 \pm 1.58^{* *}$ & $9.53 \pm 2.69^{* * *}$ \\
TG & $14.14 \pm 3.74^{* *, \#}$ & $4.86 \pm 0.18^{* * \ldots}$ & $57.67 \pm 1.28^{* * \ldots}$ & $6.76 \pm 6.87^{* * \ldots}$ \\
\hline
\end{tabular}

Notes: Data are presented as mean \pm standard deviation. $* * P<0.01$ compared with the NG and ${ }^{\# P<0.01, ~}{ }^{*} P<0.05$ compared with the MG.

Abbreviations: BUN, blood urea nitrogen; Cr, creatinine; KW/BW, kidney weight/body weight ratio; MG, model group; NG, normal control group; RBG, random blood glucose; TG, treatment group.

glomerular epithelial cell proliferation, renal interstitial inflammatory cell infiltration, partial renal tubular atrophy, and many transparent tubules. Mallory staining revealed unambiguous glomerular sclerosis and interstitial fibrosis in the DN model KKAy mice, while collagen fibers were detected in the glomerular and renal tubules of the $\mathrm{C} 57 \mathrm{BL} / 6 \mathrm{~J}$ mice (Figure 3D and E). However, treatment with BBR reversed these changes to some degree (Figure $3 \mathrm{C}$ and $\mathrm{F}$ ), which further confirms the therapeutic effect of BBR.

\section{BBR alleviates diabetes-induced podocyte injury and thickening of the glomerular basement membrane (GBM)}

To confirm whether the DN model was satisfactorily represented and the therapeutic effect of BBR, we performed the electron microscopy experiment (TEM) on kidney tissues (Figure 4). TEM observation revealed that the normal group displayed GBMs with defined structures and normal foot processes (Figure 4A), while the DN model KKAy mice showed irregular thickening of the GBM, effacement of the foot processes, and accumulation of the mesangial and renal interstitial matrix (Figure 4B). The severity of all of the morphologic changes listed above decreased to a certain extent after treatment with BBR (Figure 4C).

\section{BBR suppresses renal tubular EMT in DN model KKAy mice by upregulating the expression of $\mathrm{E}-\mathrm{Cad}$ and downregulating the expression of $\alpha$-SMA}

It is established that E-Cad is a marker of epithelial cells, while $\alpha$-SMA is a mesenchymal cell marker. The EMT is a process in which renal tubular epithelial cells lose their epithelial markers, change their morphology, become muscle fibroblasts, and obtain the mesenchymal cell marker $\alpha$-SMA, to combat injury and avoid potential apoptosis. To investigate the effects of BBR on inhibition of the EMT in DN model KKAy mice, E-Cad and $\alpha$-SMA protein expression was detected by Western blot and immunohistochemistry (Figure 5A and B). Relative to the normal group, E-Cad expression was markedly decreased in the DN model KKAy mice $(P<0.01$; Figure 5C and D), while $\alpha$-SMA expression was increased $(P<0.01$; Figure 5B and D). BBR attenuated the decreased $\alpha$-SMA expression in the DN model KKAy mice $(P<0.01$; Figure 5B and D). Moreover, E-Cad and $\alpha$-SMA mRNA expression levels were detected by ISH (Figure 5E), consistent with the results from Western blot and immunohistochemistry. The E-Cad mRNA expression level was significantly decreased in the $\mathrm{MG}$ relative to the $\mathrm{NG}$ $(P<0.01$; Figure 5F). Moreover, the $\alpha$-SMA mRNA expression was also significantly increased in the DN model KKAy mice $(P<0.01$; Figure 5F). BBR reversed the decreased $\alpha$-SMA protein expression and mRNA transcription level and increased the E-Cad protein expression and mRNA transcription level in the DN model KKAy mice $(P<0.01$; Figure 5F), which suggests that BBR can suppress the renal tubular EMT in DN model KKAy mice.

\section{BBR inhibits the activation of the Notch pathway in DN model KKAy mice}

The Notch pathway induces conformational changes of the Notch receptor (Notch1) by ligand (jagged1) and receptor binding, and the activated form of Notch (Notch intracellular domain) is released under the guidance of $\gamma$-secretase, which activates the downstream genes hes and hey after entry into the nucleus, regulating cell transdifferentiation and inducing cell differentiation. ${ }^{32}$ In our study, jagged1,

Table 3 Effects of BBR on albuminuria in diabetic nephropathy model KKAy mice $(\mathrm{mg} / 24 \mathrm{~h})$

\begin{tabular}{|c|c|c|c|c|c|}
\hline \multirow[t]{2}{*}{ Group } & \multicolumn{5}{|c|}{ Albuminuria (mg/24 h) } \\
\hline & I0th week & I4th week & I8th week & 22nd week & 26th week \\
\hline NG & $0.018 \pm 0.004$ & $0.58 \pm 0.06$ & $0.16 \pm 0.02$ & $0.33 \pm 0.06$ & $0.28 \pm 0.09$ \\
\hline MG & $2.49 \pm 0.21 * *$ & $7.37 \pm 0.21 * *$ & $\mid \mathrm{I} .8 \mathrm{I} \pm 0.99 * *$ & $18.49 \pm 2.23 * *$ & $21.01 \pm 0.94 * *$ \\
\hline TG & $2.54 \pm 0.16 * * \ldots$ & $6.85 \pm 0.32 * * \ldots$ & $10.07 \pm 0.91 * * \ldots \#$ & $15.84 \pm 0.78 * * \ldots$ & $|3.92 \pm 0.9| * * \ldots$ \\
\hline
\end{tabular}

Notes: Data are presented as mean \pm standard deviation. $* * P<0.01$ compared with the $N G$ and ${ }^{*} P<0.01$.

Abbreviations: BBR, berberine; MG, model group; NG, normal control group; TG, treatment group. 


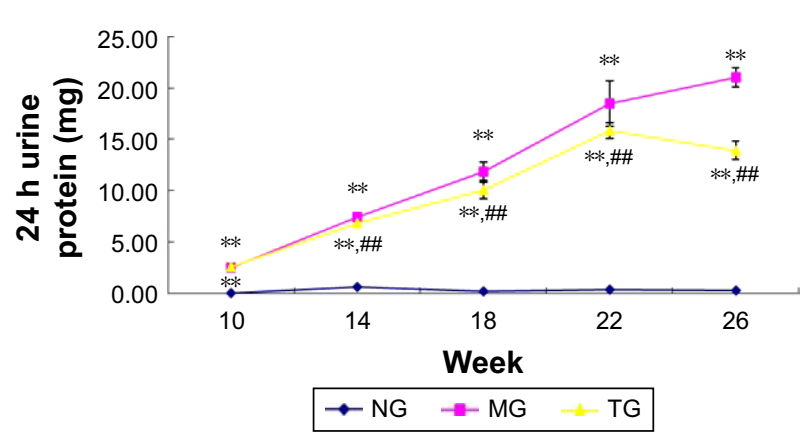

Figure 2 Effects of berberine on albuminuria in diabetic nephropathy model KKAy mice.

Notes: Data are presented as the mean \pm standard deviation. $* * P<0.01$ compared with the NG and ${ }^{\#} P<0.01$.

Abbreviations: MG, model group; NG, normal control group; TG, treatment group.

notch1, and hes 1 expression levels were detected by Western blot and immunohistochemistry (Figure 6A and B), while their mRNA levels were detected by ISH (Figure 6C). Our results show that the jagged1, notch1, and hes1 protein and mRNA levels were increased, suggesting that the Notch pathway was activated in the $\mathrm{MG}$ relative to the $\mathrm{NG}(P<0.01$; Figure 6D-F). After 16 weeks of treatment, BBR decreased the protein and mRNA levels of jagged1, notch1, and hes1 in the DN model KKAy mice $(P<0.01$; Figure 6D-F), indicating that Notch pathway activation was blocked in DN model KKAy mice.

\section{BBR inhibits the expression of snail}

Snail1 is the first member of the snail family, and it is known that snail family molecules have a role in promoting epithelial cell differentiation by direct inhibition of E-Cad expression. ${ }^{33}$ Moreover, hes1 can directly regulate snail expression in other cell lines. ${ }^{34}$ Thus, snail acts as a bridge for promoting renal tubular EMT and activating the Notch pathway in DN. Therefore, we examined the protein level of snaill by Western blot and immunohistochemistry and the mRNA level in the kidney tissues by ISH. As shown in Figure 7, the protein level of snaill was significantly increased in the DN model KKAy mice relative to the $\mathrm{C} 57 \mathrm{BL} / 6 \mathrm{~J}$ mice $(P<0.01$; Figure $7 \mathrm{~B}$ and $\mathrm{D})$. After BBR treatment, the protein level of snail1 was sharply lowered $(P<0.01$; Figure $7 \mathrm{~B}$ and D). Similarly, the snail1 mRNA level was also increased in the kidney of the DN model KKAy mice and was reduced by BBR treatment $(P<0.01$; Figure $7 \mathrm{~F})$. BBR markedly inhibited snail protein and mRNA expression, which were induced by $\mathrm{DN}$ (Figure 7E).

\section{Influence of BBR on HG-induced EMT markers in mRTECs}

To explore whether BBR suppresses the diabetes-induced renal tubular EMT and to identify the potential mechanisms, mRTECs were used as a model in vitro to evaluate the effect of BBR on the renal tubular EMT. The levels of E-Cad and $\alpha$-SMA in the mRTECs were detected by Western blot and RT-PCR. As shown in Figure 8, the reduction of E-Cad protein expression in $\mathrm{HG}$ conditions was accompanied by an increase in $\alpha$-SMA protein expression, which was significantly different from the $\mathrm{NG}$, and confirmed that $\mathrm{HG}$ promotes EMT development in mRTECs (Figure 8A and B). However, the HG-induced EMT was suppressed after treating mRTECs with BBR, which demonstrated that BBR could reduce $\alpha$-SMA expression. Additionally, the inhibitory effect of BBR on HG-stimulated EMT was further confirmed by RT-PCR; HG treatment in the cells for $48 \mathrm{~h}$ caused a clear
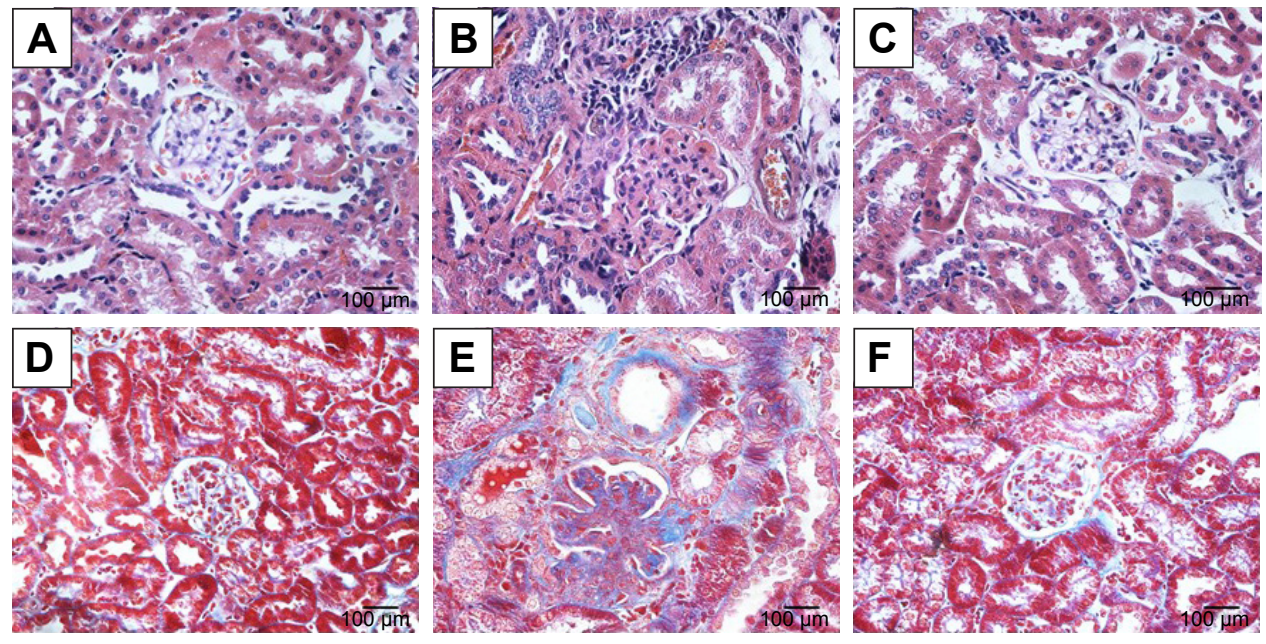

Figure 3 Effect of berberine on the morphologic changes in the kidneys of DN model KKAy mice.

Notes: (A-C) HE staining, 400×. (A) The NG, (B) the MG, and (C) the TG. (D-F) Mallory staining, 400×. (D) The NG, (E) the MG, and (F) the TG.

Abbreviations: DN, diabetic nephropathy; HE, hematoxylin and eosin; MG, model group; NG, normal control group; TG, treatment group. 

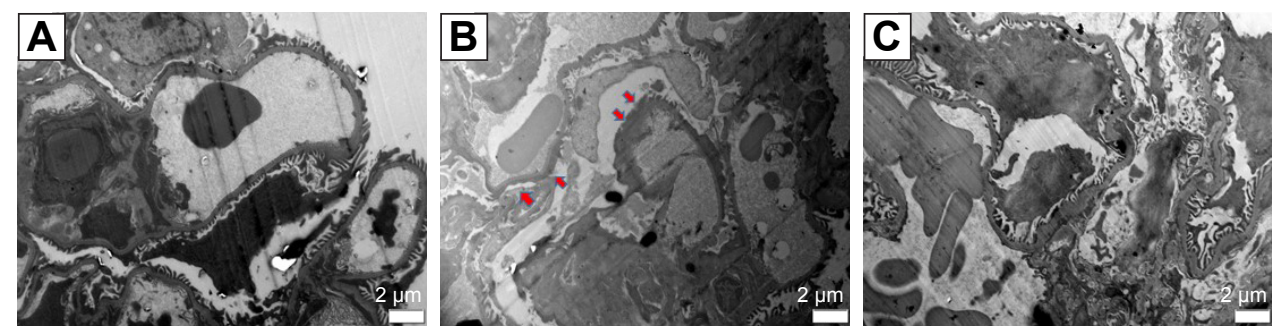

Figure 4 Berberine alleviated diabetes-induced podocyte injury and thickening of the glomerular basement membrane.

Notes: Representative fields of podocyte foot processes under electron microscopy (scale bars: $2 \mu \mathrm{m}$, red arrows indicate podocyte foot process effacement). (A) The normal control group, (B) the model group, and $(\mathbf{C})$ the treatment group.

increase leading to an obvious increase in the RNA level of $\alpha$-SMA and decrease in the RNA level of E-Cad (Figure $8 \mathrm{C}$ ), which could be attenuated by treatment with $30 \mu \mathrm{M}$ BBR; this was consistent with the results of the Western blot. These data demonstrate that BBR reverses HG-stimulated EMT in mRTECs.

\section{BBR inhibits activation of the Notch pathway in HG-exposed mRTECs}

In our in vitro study, jagged1, notch1, and hes1 expression levels were detected by Western blot (Figure 9A and B) and the mRNA level was detected by RT-PCR (Figure 9C). Our results showed that the expression of jagged1, notch1, and hes1
A

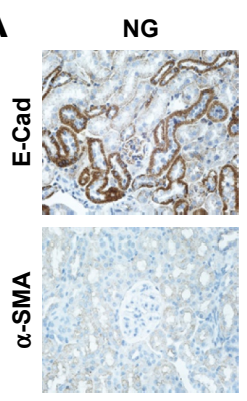

B

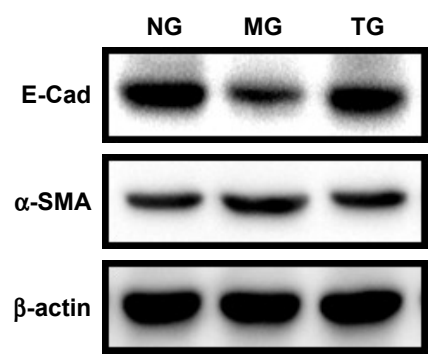

E

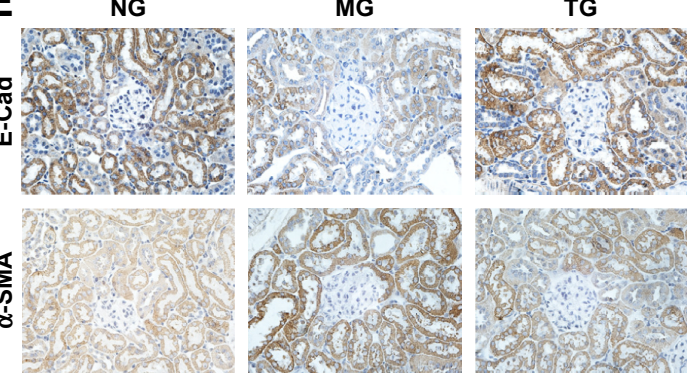

C
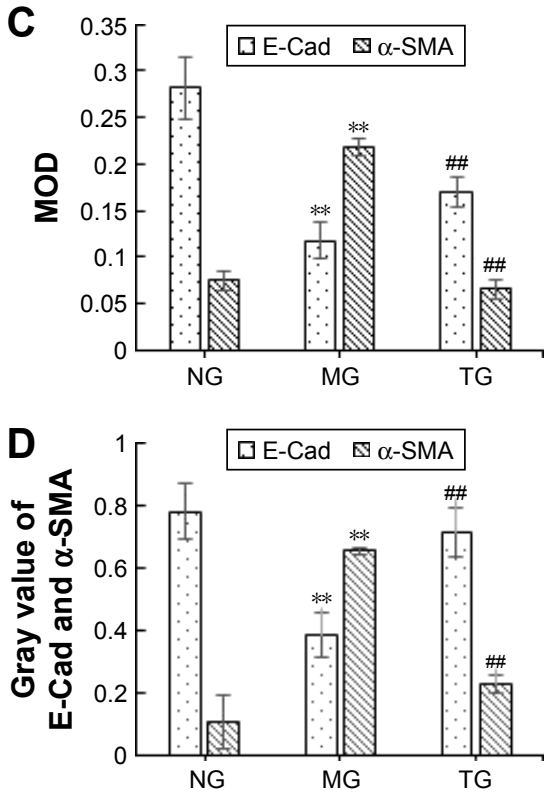

$\mathbf{F}$

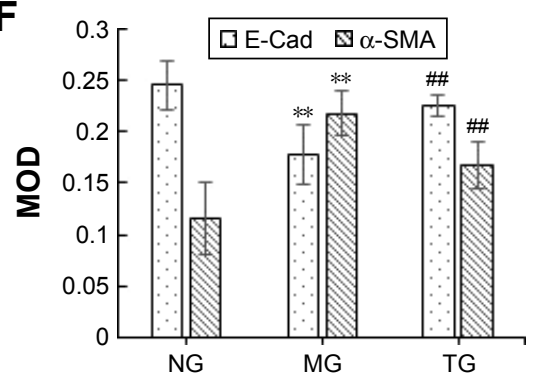

Figure 5 Berberine suppresses renal tubular epithelial-to-mesenchymal transition in DN model KKAy mice by upregulating the expression of E-Cad and downregulating the expression of $\alpha$-SMA.

Notes: (A) Representative photographs of immunohistochemistry for E-Cad and $\alpha$-SMA in DN model KKAy mice, 400×. (B) Representative band of E-Cad and $\alpha$-SMA proteins by Western blot in DN model KKAy mice. (C) Comparison of MOD of E-Cad and $\alpha$-SMA proteins in DN model KKAy mice. (D) Comparison of the gray value of E-Cad and $\alpha$-SMA proteins in DN model KKAy mice $(n=3)$. (E) Representative photographs of in situ hybridization for E-Cad and $\alpha$-SMA in DN model KKAy mice, 400X. (F) Comparison of MOD of E-Cad and $\alpha$-SMA mRNA in DN model KKAy mice. **P $<0.01$, NG and ${ }^{\#} P<0.01$.

Abbreviations: DN, diabetic nephropathy; E-Cad, E-cadherin; MG, model group; MOD, mean optical density; NG, normal control group; TG, treatment group. 
A
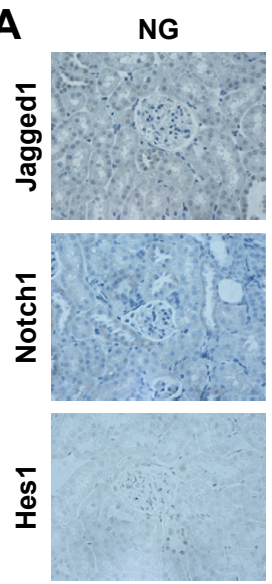

B
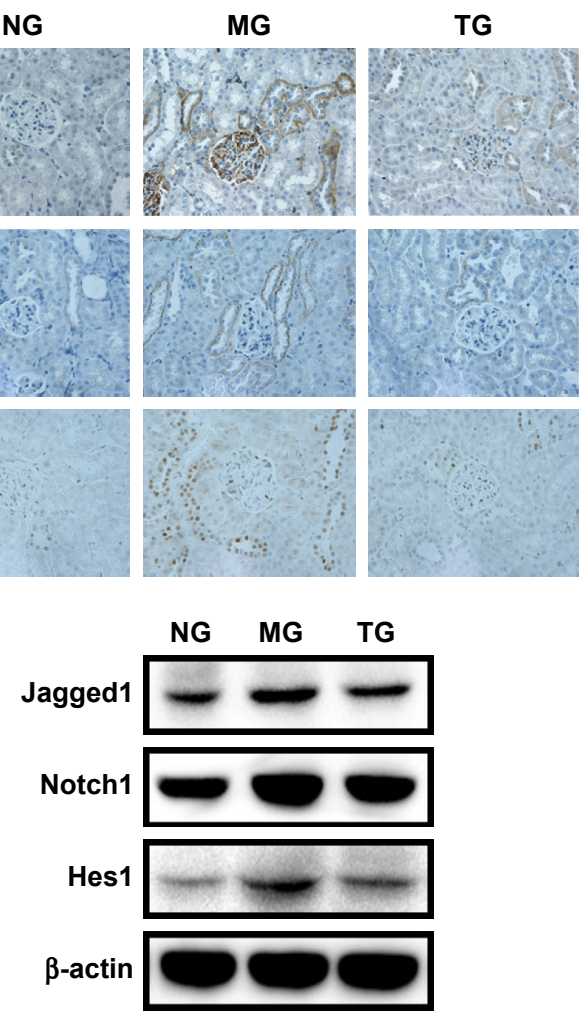

C
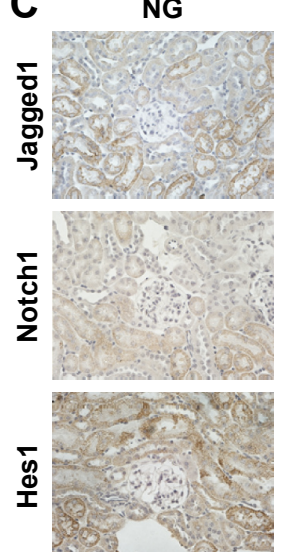

MG
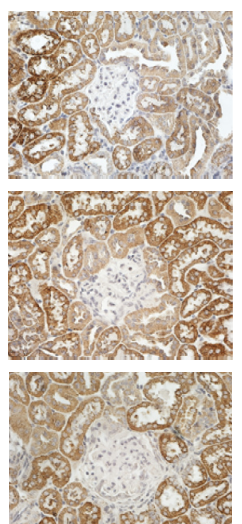
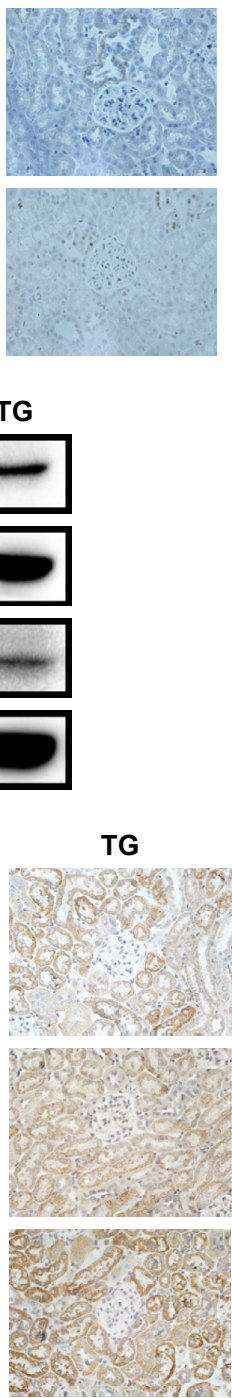

D

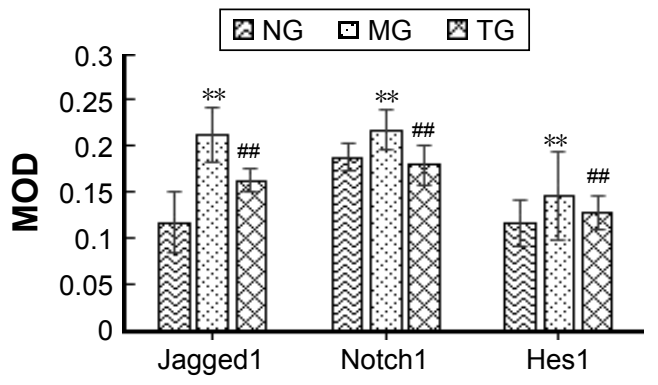

E

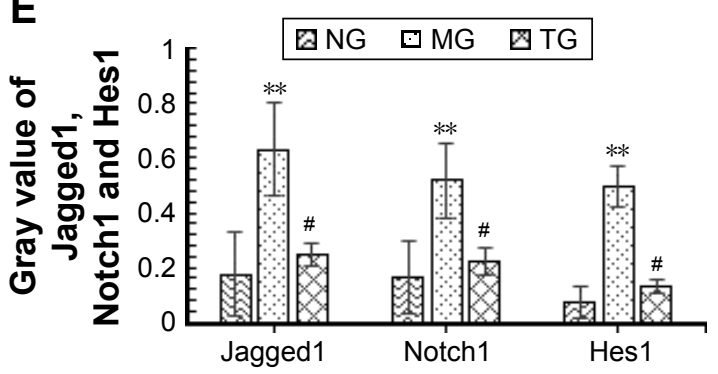

$\mathbf{F}$

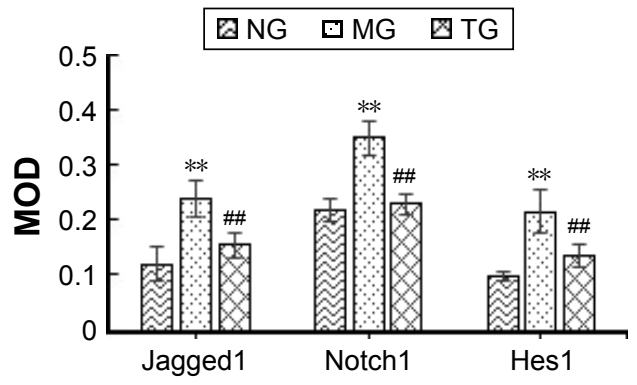

Figure 6 Berberine inhibits activation of the Notch pathway in DN model KKAy mice.

Notes: (A) Representative photographs of immunohistochemistry for JaggedI, Notch I, and HesI in DN model KKAy mice, 400×. (B) Representative band of Jagged I, Notch I, and Hes I proteins by Western blot in DN model KKAy mice. (C) Representative photographs of in situ hybridization for Jagged I, notch I, and Hes I in DN model KKAy mice, 400x. (D) Comparison of MOD of Jagged I, notch I, and Hes I proteins in DN model KKAy mice. (E) Comparison of the gray value of Jagged I, Notch I, and Hes I proteins in DN model KKAy mice $(n=3)$. ${ }^{* * P}<0.01$ and ${ }^{\#} P<0.01, * P<0.05$ compared with the MG.

Abbreviations: DN, diabetic nephropathy; MG, model group; MOD, mean optical density; NG, normal control group; TG, treatment group.

was lower in the mRTECs in the NG than in the HG group (Figure 9). HG significantly increased the jagged1, notch1, and hes1 expression and the mRNA level (Figure 9C). BBR markedly decreased the jagged1, notch1, and hes 1 protein expression induced by HG $(P<0.05$; Figure $9 \mathrm{~A}$ and $\mathrm{B})$. Moreover, BBR slightly decreased the jagged1, notch1, and hes1 mRNA expression induced by $\mathrm{HG}(P<0.05$; Figure $9 \mathrm{C})$. The above results consistently indicate that BBR treatment can inhibit the activation of the Notch pathway induced by HG.

\section{BBR inhibits HG-induced expression of snail I}

Similarly, snail1 protein and mRNA expression of mRTECs were detected by Western blot and RT-PCR, respectively, in our in vitro study. DAPT is a $\gamma$-secretase inhibitor and an indirect inhibitor of the Notch pathway. To further clarify the relationship between the Notch pathway and snail1, in the DAPT group, the cells were treated with $30 \mathrm{mM} / \mathrm{L}$ glucose after pretreatment with $10 \mu \mathrm{M} / \mathrm{L}$ DAPT for $40 \mathrm{~min}$. 
A

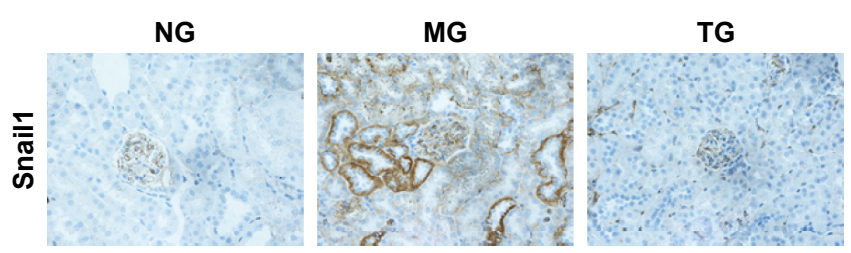

C

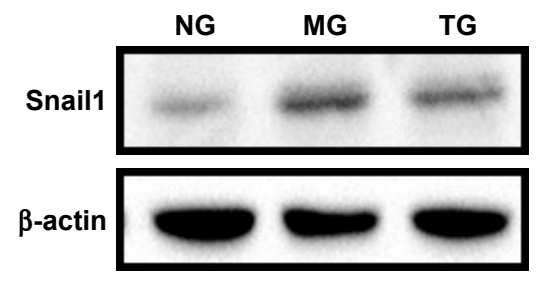

E

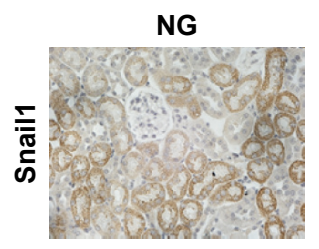

MG

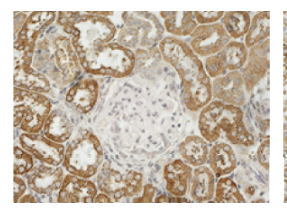

TG

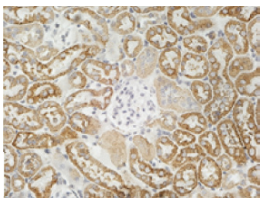

B
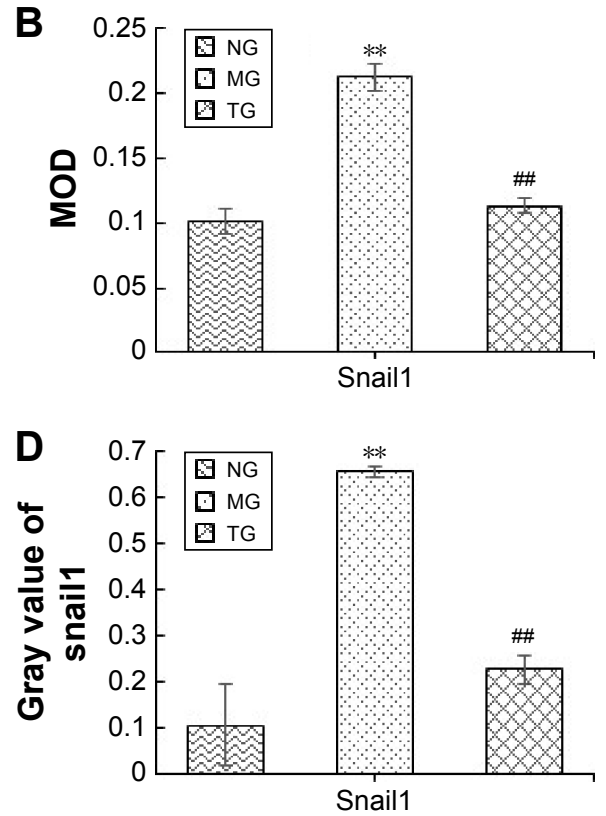

F

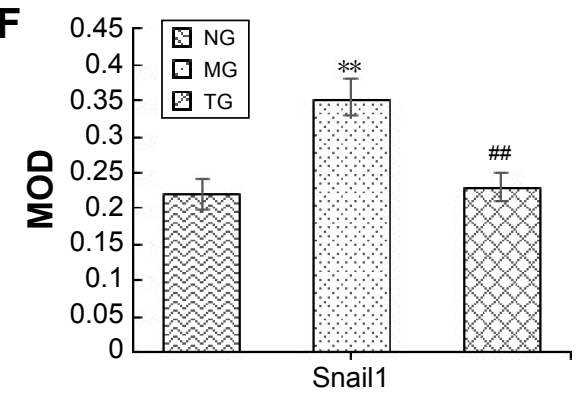

Figure 7 Berberine inhibits the expression of snaill.

Notes: (A) Representative photographs of immunohistochemistry of snaill in DN model KKAy mice. 400× magnification. (B) Comparison of MOD of snaill protein in DN model KKAy mice. (C) Representative band of snail l protein by Western blot in DN model KKAy Mice. (D) Comparison of the gray value of snail I protein in DN model KKAy mice $(n=3)$. (E) Representative photographs of in situ hybridization for snail I in DN model KKAy mice. 400× magnification. (F) Comparison of MOD of snail I mRNA in DN model KKAy mice. ${ }^{* *} P<0.01$ compared with the $\mathrm{NC}$ and ${ }^{\#} P<0.01$ compared with the MG.

Abbreviations: DN, diabetic nephropathy; MG, model group; MOD, mean optical density; NG, normal control group; TG, treatment group.

Our results showed that snaill protein and mRNA expression were significantly increased in the $\mathrm{HG}$ group relative to the NG $(P<0.05$; Figure 10A-C); also, BBR markedly inhibited snaill protein and mRNA expression induced by $\mathrm{HG}$ $(P<0.05$; Figure 10A-C). Moreover, the protein and mRNA expression level of snaill were also decreased in the DAPT group $(P<0.05$; Figure $10 \mathrm{~A}-\mathrm{C})$, which implies that the snail 1 expression is indeed regulated by the Notch pathway.

\section{Discussion}

The incidence of diabetes is increasing yearly because of changes in lifestyle and dietary structure. At present, $7 \%$ of Chinese adults are DM patients, as many as 0.92 billion. ${ }^{35}$ Among patients who have had diabetes for a period of 10-20 years, approximately one-third will develop DN. ${ }^{36}$ The prevalence rate of $\mathrm{DN}$ in our country is increasing, and middle-aged and elderly people account for a large portion, with the patient group having a tendency to become younger. $\mathrm{DN}$ is still one of the main causes of chronic renal failure, ${ }^{37}$ and its treatment is far more complicated than that of general kidney disease, with a poor prognosis. Currently, western medicine mainly works to regulate the blood glucose, blood lipids, and blood pressure and to improve the microcirculation to control the disease; however, due to the lack of effective drug treatment, it is difficult to prevent the development of the disease. ${ }^{38}$ The etiology of DN is complex, and its pathogenesis, which involves many factors, is still unclear. Therefore, the focus of the current study is to identify how to effectively prevent and control DN and how to prevent further deterioration of renal function. Renal interstitial fibrosis is the main pathological change in the progression of chronic renal disease to end-stage renal failure. In the present study, it was found that more than one-third of the interstitial fibroblasts were derived from the EMT of renal tubular epithelial cells, 


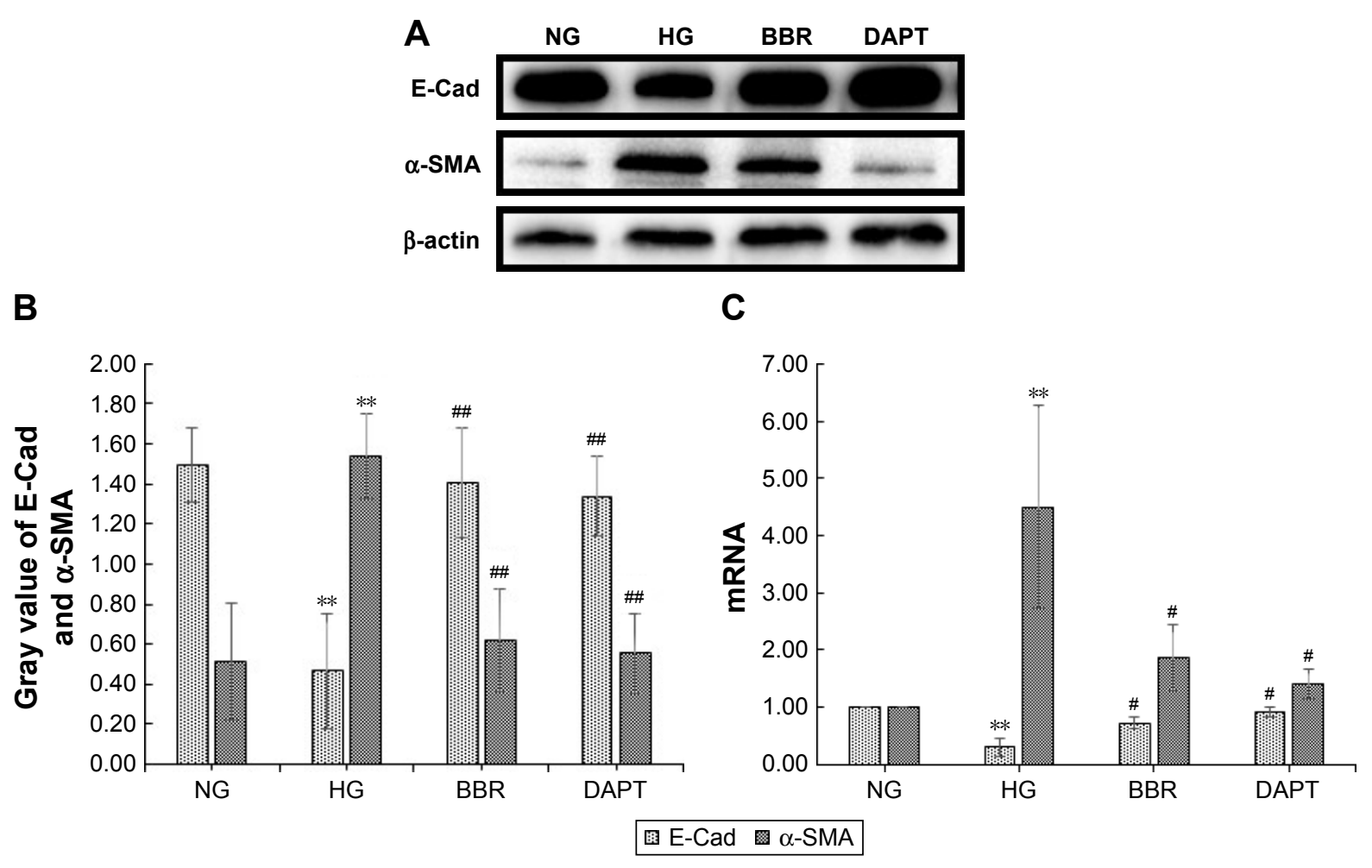

Figure 8 Influence of BBR on HG-induced epithelial-to-mesenchymal transition markers in mRTECs.

Notes: (A) Representative band of E-Cad and $\alpha$-SMA proteins by Western blot in mRTECs. (B) Comparison of the gray value of E-Cad and $\alpha$-SMA proteins in mRTECs $(n=3)$. (C) Comparison of the mRNA levels of E-Cad and $\alpha$-SMA by reverse transcription polymerase chain reaction in $m R T E C s(n=3)$. $* * P<0.0 I$ compared with the NG and ${ }^{\# P}<0.01,{ }^{\#}<<0.05$ compared with the HG group.

Abbreviations: BBR, berberine; DAPT, $N$-[N-(3,5-difluorophenacetyl)-L-alanyl]-S-phenylglycine t-butyl ester; E-Cad, E-cadherin; HG, high glucose; mRTECs, mouse renal tubular epithelial cells; NG, normal control group.

which may be one of the most important mechanisms leading to the occurrence of renal interstitial fibrosis. Inhibiting the EMT of renal tubular epithelial cell differentiation is an important target for delaying the progression of DN.

In the process of renal fibrosis in DN, the EMT is a unique event in renal tubular epithelial cells. As a key factor in the loss of renal units in DN, the renal tubular epithelial EMT is reversible under certain conditions and has become a focus and a breakthrough area in DN research in recent years, with a high research value. ${ }^{5}$

In this experiment, KKAy mice were used as the animal model, and the DN model was established by continuous feeding of the KKAy mice with KK mouse feed. Mice of the KK strain develop diabetes of polygenic origin, and KK. $\mathrm{Cg}-\mathrm{Ay} / \mathrm{J}$ heterozygotes develop hyperglycemia, hyperinsulinemia, glucose intolerance, and obesity by 8 weeks of age. Pancreatic islets are hypertrophied, and the $\beta$-cells are degranulated. Both the fat and lean tissue mass are increased relative to nonobese mice, with fat accounting for 30\%-35\% of the total body weight. ${ }^{39}$ There are many advantages of this model, including small individual differences, short formation time, strong operability, and favorable repeatability.
The multiple factors leading to its pathogenesis are similar to human type $2 \mathrm{DM}$ and simulate the human diet and lifestyle. Domestic and foreign research has shown that the blood glucose and $24 \mathrm{~h}$ urinary protein excretion rate of KKAy mice are increased after 8 weeks of a high-fat diet and that the kidney weight and body weight are significantly greater than those in the control group. $.^{40} \mathrm{C} 57 \mathrm{BL} / 6 \mathrm{~J}$ mice are black and exhibit gene homology with KK mice as an NG. ${ }^{41}$ On this basis, we extended the course of DM in KKAy mice, which made the model much more similar to the human DN process. Relative to the NG, the body weight, blood glucose, blood serum $\mathrm{Cr}$, and BUN of the mice in the MG increased significantly and the $24 \mathrm{~h}$ urinary protein excretion increased gradually with time. HE staining showed that a variety of $\mathrm{DN}$-induced changes in renal morphology were detected in the pathology results from the $\mathrm{MG}$, including glomerular hypertrophy, mesangial cell proliferation, vacuolar degeneration in the renal tubular epithelial cells, and inflammatory cell infiltration in the renal interstitium. Mallory staining revealed glomerular sclerosis and renal interstitial fibrosis in the MG. Many collagen fibers were found in the glomerular and renal tubular interstitia. Furthermore, ultrastructural analyses 


\section{A}
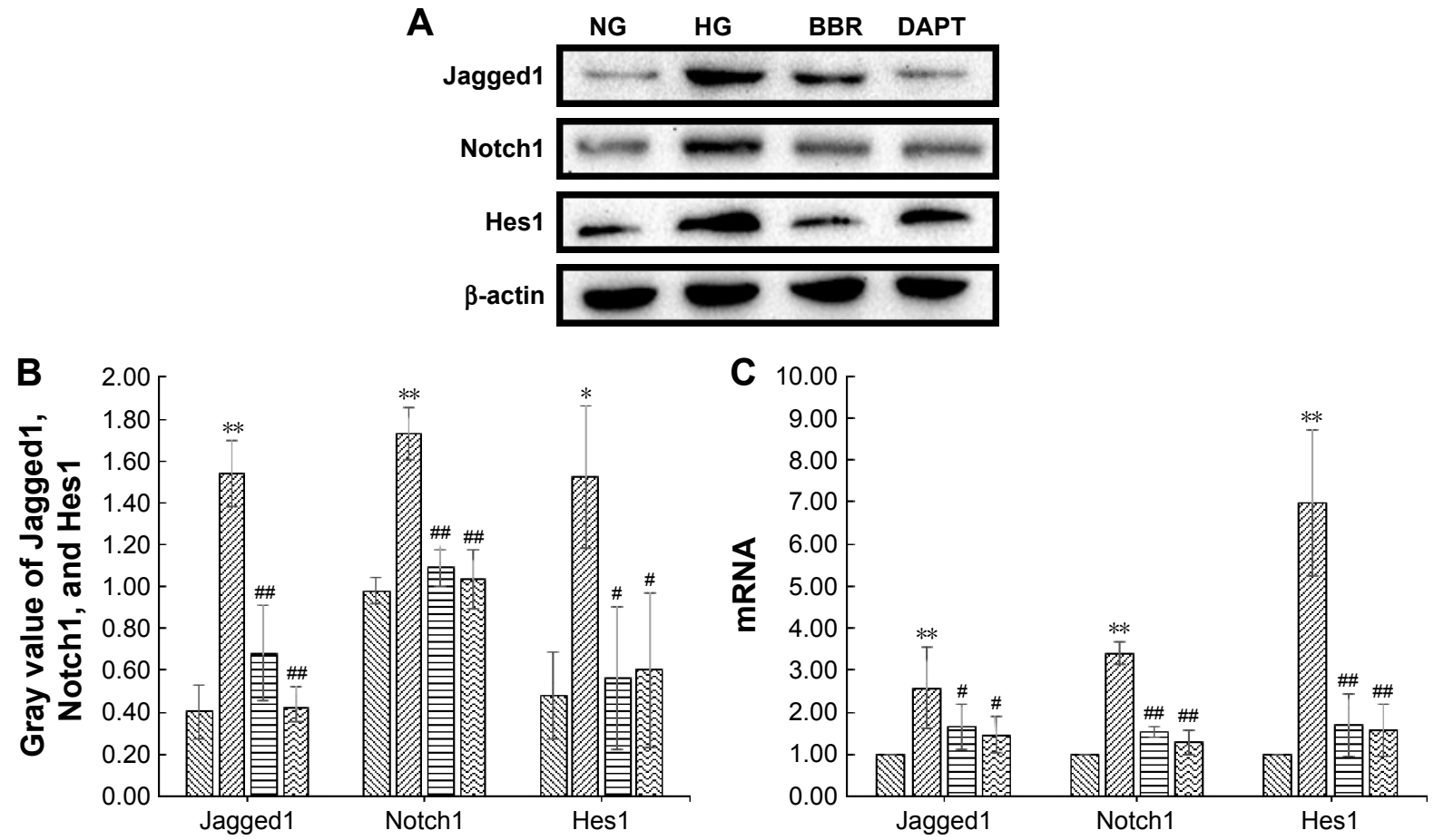

QNG $\quad$ I H

Figure 9 BBR inhibits activation of the Notch pathway in HG-exposed mRTECs.

Notes: (A) Representative band of JaggedI, Notchl, and Hesl proteins by Western blot in mRTECs. (B) Comparison of the gray value of JaggedI, Notchl, and Hes I proteins in mRTECs $(n=3)$. (C) Comparison of the mRNA levels of Jaggedl, Notchl, and Hesl by reverse transcription polymerase chain reaction in $m R T E C s$ ( $n=3$ ). $* * P<0.01, * P<0.05$ compared with the $N G$ and $\# P<0.01$, $\# P<0.05$ compared with $H G$ group.

Abbreviations: BBR, berberine; DAPT, $N$-[N-(3,5-difluorophenacetyl)-L-alanyl]-S-phenylglycine $t$-butyl ester; HG, high glucose; mRTECs, mouse renal tubular epithelial cells; NG, normal control group.
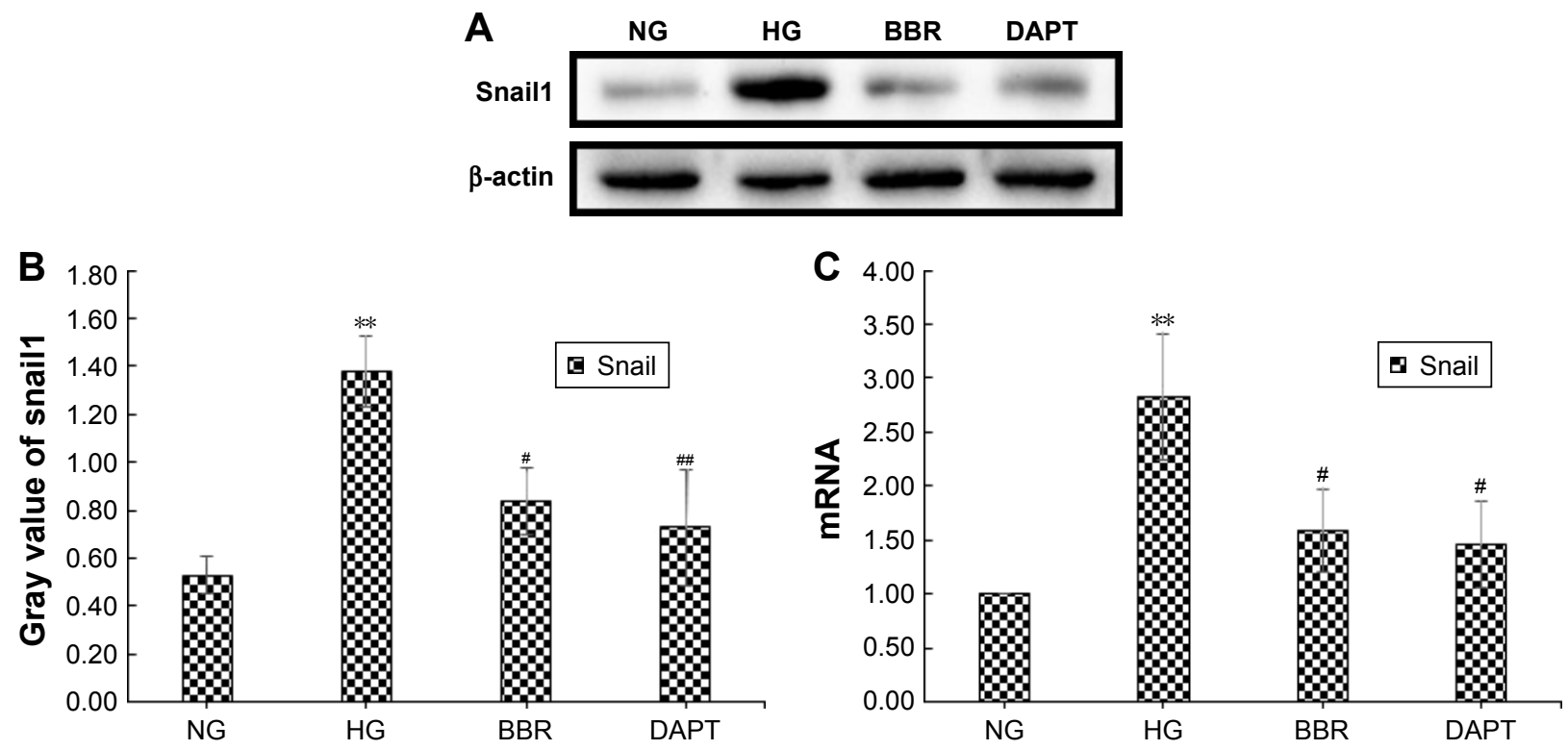

Figure 10 BBR inhibits the HG-induced expression of snaill.

Notes: (A) Representative band of snaill protein by Western blot in mRTECs. (B) Comparison of the gray value of snail I protein in $m R T E C s$ ( $n=3$ ). (C) Comparison of the mRNA level of snaill protein by reverse transcription polymerase chain reaction in mRTECs $(n=3)$. ${ }^{* * P}<0.01 \mathrm{NG}$ and ${ }^{\#} P<0.0 \mathrm{I}$, ${ }^{*} P<0.05$ compared with the model group. Abbreviations: BBR, berberine; DAPT, $\mathrm{N}$-[N-(3,5-difluorophenacetyl)-L-alanyl]-S-phenylglycine $t$-butyl ester; HG, high glucose; mRTECs, mouse renal tubular epithelial cells; NG, normal control group. 
revealed irregular thickening of the GBM and effacement of the foot processes. In sum, the KKAy mouse model of DN was fully successful, and we successfully replicated the model of renal interstitial fibrosis.

In China, TCM has long been widely used to treat diabetes and its complications. ${ }^{42}$ TCM has many advantages over the conventional medical approaches in the prevention of diabetic complications because of less toxicity and/or fewer side effects. ${ }^{43-45}$ Xiao et al ${ }^{46}$ reviewed more than 2,000 published papers and established that Chinese herbal medicine was an effective and safe method for reducing urinary proteins in patients with DN. TCM has certain advantages in the treatment of $\mathrm{DN}$, and it is of great significance to further study the mechanisms of preventing and treating DN. BBR, an effective compound of herbal TCM, includes Coptis chinensis, Hydrastis canadensis, Berberis aristata, Berberis aquifolium, and Arcangelisia flava. ${ }^{3} \mathrm{BBR}$ is endowed with several pharmacologic activities, including antimicrobial, glucose- and cholesterol-lowering, antitumoral, and immunomodulatory properties. ${ }^{47} \mathrm{BBR}$ is used to treat a variety of diseases such as type 2 diabetes, hyperlipidemia, heart diseases, cancers, and inflammation. BBR has been reported to have apoptotic activity in a variety of human tumor cell lines, including hepatoma, colon cancer, lung cancer, breast cancer, and leukemia cell lines. BBR may act on cancer cell DNA, DNA replication enzymes (eg, telomerase and DNA topoisomerase), matrix metalloproteinases in the mitochondria, the $\mathrm{Bcl} 2 / \mathrm{Bax}$ ratio, p53, and apoptosis inducing factor. ${ }^{48,49}$ In the clinic, BBR is often used as a treatment for patients with DN in China. Evidence is available that BBR protects human renal proximal tubular cells from hypoxia/ reoxygenation injury by inhibiting endoplasmic reticulum and mitochondrial stress. ${ }^{31}$ Zhang et $\mathrm{al}^{26}$ have recently demonstrated that BBR can ameliorate tubulointerstitial fibrosis in DN by activating the Nrf2 pathway and inhibiting TGF- $\beta$ / Smad/EMT signaling activity.

In the present study, accumulating evidence from metabolic biochemical parameters and morphologic changes indicates that $\mathrm{BBR}$ is beneficial for the recovery of renal function in DN.

In the BBR TG, the body weight of DN mice was low, the $24 \mathrm{~h}$ urinary protein excretion decreased, and the levels of urea nitrogen and $\mathrm{Cr}$ were decreased. Renal pathological injury was significantly reduced. Most importantly, BBR inhibited renal tubular epithelial EMT and renal interstitial fibrosis.

However, the renal protective function of BBR is multitargeted, and its mechanism is relatively complex. Little is known about the role and underlying mechanism of BBR in interstitial tubules, particularly in DN. In the present study, it was confirmed that DN can induce changes in EMT markers by decreasing the epithelial marker E-Cad and increasing $\alpha$-SMA relative to that in the control. In vitro experiments also confirmed this result. Moreover, BBR pretreatment may protect against EMT and decrease $\alpha$-SMA and increase E-Cad expression in vivo and in vitro, which are associated with the EMT. Furthermore, this experiment was aimed to explore the molecular mechanism through which BBR inhibits the EMT in DN. The Notch pathway is known to be linked to DN. Russell et al ${ }^{50}$ observed that jagged 1 increased significantly in primary muscle fibroblasts. Bonegio and Susztak $^{10}$ reported hyperplasia of the extracellular matrix and further development of renal tubular interstitial fibrosis related to the reactivation of the Notch signaling pathway in DN. This concept is further supported by the fact that DN renal tubular interstitial fibrosis can be improved and inhibited by treatment with Notch signaling pathway inhibitors. The researchers performing these studies have stressed that the notch1/jagged1 signaling pathway may be the key to DN development. ${ }^{10}$ The experiment of Sassoli et $\mathrm{al}^{51}$ showed that the cell changes induced by Notch pathway activation were consistent with the change in the cell EMT. Similar experiments also suggest that Notch signaling and renal fibrosis are closely related. However, multiple studies show that the EMT mediated by the Notch pathway is not a complete EMT process. On the one hand, it promotes epithelial cell differentiation; on the other hand, it limits cells from continuing their differentiation. Ultimately, only a few renal tubular epithelial cells that pass through the basement membrane become fibroblasts. As an important cytokine in the renal tubular epithelial cell EMT, snail plays a key role in the promotion of the EMT. Further study has revealed that the expression of snail protein in renal tubular epithelial cells is downregulated after blocking the Notch pathway and that the formation of E-Cad is accelerated, followed by the downregulation of $\alpha$-SMA and the renal tubular epithelial EMT. ${ }^{52-55}$ Recent investigations have also shown that the Notch pathway can induce the renal tubular epithelial EMT by activating snail. ${ }^{56}$ In sum, the Notch/snail pathway is an important molecular mechanism in DN renal interstitial fibrosis. The Notch/ snail pathway is also an important node of intervention of DN renal interstitial fibrosis and an important drug target for regulating interstitial fibrosis. In our study, the amount of jagged1, notch1, hes1, and snail1 expression was higher in the DN MG of KKAy mice than in the NG, indicating 
the activation of the Notch/snail pathway. Additionally, treatment with BBR significantly reduced jagged1, notch1, hes1, and snail1 mRNA expression, which suggested that BBR might play a role in downregulating the Notch/snail pathway at the transcriptional level. Our Western blot and immunohistochemistry analyses confirmed that the jagged1, notch1, hes1, and snail1 proteins were upregulated in the MG and downregulated in the NG, which inhibited activation of the Notch/snail pathway and improved the renal conditions. Moreover, the study of the mechanism by which BBR prevented HG-induced EMT events is important because BBR can inhibit the HG-induced expression of Notch pathway members and snail 1 in mRTECs. More importantly, treatment with DAPT also inhibited the HG-induced expression of the Notch/snail pathway and EMT, which demonstrated that the Notch pathway can induce renal tubular epithelial EMT by activating snail. These findings demonstrate that BBR ameliorates renal injury in diabetic mice by inhibiting the Notch/snail pathway and provide further evidence for the effectiveness of BBR in the treatment of DN.

\section{Conclusion}

In conclusion, we speculated that BBR administration could suppress renal tubular epithelial EMT, reduce renal interstitial fibrosis, and delay the progression of DN. BBR might have a pleiotropic effect on the Notch/snail pathway both indirectly and directly, which may be a mechanism underlying the protective effect of BBR on DN model KKAy mice. Even though we confirmed our assumption, there may still be certain limitations to our research. To rule out a possible specific effect of BBR, we should add a group of control mice treated with BBR; under this scenario, we would need to more thoroughly explore this subject and address these limitations in subsequent experiments. Although BBR has a pleiotropic effect on tubulointerstitial fibrosis in DN, clinical trials are still necessary for further confirmation.

\section{Acknowledgment}

This study was supported by the National Natural Science Foundation of China (number 81373831).

\section{Disclosure}

The authors report no conflicts of interest in this work.

\section{References}

1. Shi Y, Hu FB. The global implications of diabetes and cancer. Lancet. 2014;383(9933):1947-1948.
2. Kanwar YS, Sun L, Xie P, Liu FY, Chen S. A glimpse of various pathogenetic mechanisms of diabetic nephropathy. Annu Rev Pathol. 2011; 6:395-423.

3. Motawi TK, Ahmed SA, Hamed MA, Elmaraghy SA, Aziz WM. Combination of melatonin and certain drugs for treatment of diabetic nephropathy in streptozotocin-induced diabetes in rats. Diabetol Int. 2016;7(4):413-424.

4. He J, Xu Y, Koya D, Kanasaki K. Role of the endothelial-tomesenchymal transition in renal fibrosis of chronic kidney disease. Clin Exp Nephrol. 2013;17(4):488-497.

5. Powell DW, Bertram CC, Cummins TD, et al. Renal tubulointerstitial fibrosis in OVE26 type 1 diabetic mice. Nephron Exe Nephrol. 2008; 111(1):e11-e19.

6. Morrissey J, Guo G, Moridaira K, et al. Transforming growth factor-beta induces renal epithelial jagged-1 expression in fibrotic disease. $J \mathrm{Am}$ Soc Nephrol. 2002;13(6):1499-1508.

7. Murea M, Park JK, Sharma S, et al. Expression of Notch pathway proteins correlates with albuminuria, glomerulosclerosis, and renal function. Kidney Int. 2010;78(5):514-522.

8. Sharma S, Sirin Y, Susztak K. The story of Notch and chronic kidney disease. Curr Opin Nephrol Hypertens. 2011;20(1):56-61.

9. Bielesz B, Sirin Y, Si H, et al. Epithelial notch signaling regulates interstitial fibrosis development in the kidneys of mice and humans. J Clin Invest. 2010;120(11):4040-4054.

10. Bonegio R, Susztak K. Notch signaling in diabetic nephropathy. Exp Cell Res. 2012;318(9):986-992.

11. Katoh M, Katoh M. Comparative genomics on SNAI1, SNAI2, and SNAI3 orthologs. Oncol Rep. 2005;14(4):1083-1086.

12. Paznekas WA, Okajima K, Schertzer M, Wood S, Jabs EW. Genomic organization, expression, and chromosome location of the human SNAIL gene (SNAI1) and a related processed pseudogene (SNAI1P). Genomics. 1999;62(1):42-49.

13. Peinado H, Ballestar E, Esteller M, Cano A. Snail mediates E-cadherin repression by the recruitment of the $\operatorname{Sin} 3 \mathrm{~A} /$ histone deacetylase 1 (HDAC1)/HDAC2 complex. Mol Cell Biol. 2004;24(1):306-319.

14. Herranz N, Pasini D, Diaz VM, et al. Polycomb complex 2 is required for E-cadherin repression by the Snail1 transcription factor. Mol Cell Biol. 2008;28(15):4772-4781.

15. Matsuno Y, Coelho AL, Jarai G, Westwick J, Hogaboam CM. Notch signaling mediates TGF-beta1-induced epithelial-mesenchymal transition through the induction of Snai1. Int J Biochem Cell Biol. 2012; 44(5):776-789.

16. Tuttle KR, Bakris GL, Toto RD, McGill JB, Hu K, Anderson PW. The effect of ruboxistaurin on nephropathy in type 2 diabetes. Diabetes Care. 2005;28(11):2686-2690.

17. Mann JF, Green D, Jamerson K, et al. Avosentan for overt diabetic nephropathy. J Am Soc Nephrol. 2010;21(3):527-535.

18. Pazdro R, Burgess JR. The role of vitamin $\mathrm{E}$ and oxidative stress in diabetes complications. Mech Ageing Dev. 2010;131(4):276-286.

19. Jin H, Piao SG, Jin JZ, et al. Synergistic effects of leflunomide and benazepril in streptozotocin-induced diabetic nephropathy. Nephron Exp Nephrol. 2014;126(3):148-156.

20. Tone A, Shikata K, Sasaki M, et al. Erythromycin ameliorates renal injury via anti-inflammatory effects in experimental diabetic rats. Diabetologia. 2005;48(11):2402-2411.

21. Leng SH, Lu FE, Xu LJ. Therapeutic effects of berberine in impaired glucose tolerance rats and its influence on insulin secretion. Acta Pharmacol Sin. 2004;25(4):496-502.

22. Domitrovic R, Cvijanovic O, Pernjak-Pugel E, Skoda M, Mikelic L, Crncevic-Orlic Z. Berberine exerts nephroprotective effect against cisplatin-induced kidney damage through inhibition of oxidative/ nitrosative stress, inflammation, autophagy and apoptosis. Food Chem Toxicol. 2013;62:397-406.

23. Zhang M, Lv X, Li J, et al. Sodium caprate augments the hypoglycemic effect of berberine via AMPK in inhibiting hepatic gluconeogenesis. Mol Cell Endocrinol. 2012;363(1-2):122-130. 
24. Zhang Y, Peng F, Gao B, Ingram AJ, Krepinsky JC. High glucoseinduced RhoA activation requires caveolae and PKCbeta1-mediated ROS generation. Am J Physiol Renal Physiol. 2012;302(1):F159-F172.

25. Wang FL, Tang LQ, Yang F, Zhu LN, Cai M, Wei W. Renoprotective effects of berberine and its possible molecular mechanisms in combination of high-fat diet and low-dose streptozotocin-induced diabetic rats. Mol Biol Rep. 2013;40(3):2405-2418.

26. Zhang X, He H, Liang D, et al. Protective effects of berberine on renal injury in streptozotocin (STZ)-induced diabetic mice. Int J Mol Sci. 2016; 17(8):E1327.

27. Lan T, Liu W, Xie X, et al. Berberine suppresses high glucose-induced TGF-betal and fibronectin synthesis in mesangial cells through inhibition of sphingosine kinase 1/AP-1 pathway. Eur J Pharmacol. 2012;697(1-3): $165-172$.

28. Jiang Q, Liu P, Wu X, et al. Berberine attenuates lipopolysaccharideinduced extracelluar matrix accumulation and inflammation in rat mesangial cells: involvement of NF-kappaB signaling pathway. Mol Cell Endocrinol. 2011;331(1):34-40.

29. Liu W, Tang F, Deng Y, et al. Berberine reduces fibronectin and collagen accumulation in rat glomerular mesangial cells cultured under high glucose condition. Mol Cell Biochem. 2009;325(1-2):99-105.

30. Liu W, Zhang X, Liu P, et al. Effects of berberine on matrix accumulation and NF-kappa B signal pathway in alloxan-induced diabetic mice with renal injury. Eur J Pharmacol. 2010;638(1-3):150-155.

31. Yu W, Sheng M, Xu R, et al. Berberine protects human renal proximal tubular cells from hypoxia/reoxygenation injury via inhibiting endoplasmic reticulum and mitochondrial stress pathways. J Transl Med. 2013;11(1):24

32. McCright B. Notch signaling in kidney development. Curr Opin Nephrol Hypertens. 2003;12(1):5-10.

33. Olmeda D, Jorda M, Peinado H, Fabra A, Cano A. Snail silencing effectively suppresses tumour growth and invasiveness. Oncogene. 2007; 26(13):1862-1874.

34. Sumual S, Saad S, Tang O, et al. Differential regulation of Snail by hypoxia and hyperglycemia in human proximal tubule cells. Int $J$ Biochem Cell Biol. 2010;42(10):1689-1697.

35. Jia W, Tong N. Diabetes prevention and continuing health-care reform in China. Lancet Diabetes Endocrinol. 2015;3(11):840-842.

36. Farag YM, Al Wakeel JS. Diabetic nephropathy in the Arab Gulf countries. Nephron Clin Pract. 2011;119(4):c317-c322; discussion c322-c313.

37. Low WY, Lee YK, Samy AL. Non-communicable diseases in the Asia-Pacific region: prevalence, risk factors and community-based prevention. Int J Occup Med Environ Health. 2015;28(1):20-26.

38. Sun GD, Li CY, Cui WP, et al. Review of herbal traditional Chinese medicine for the treatment of diabetic nephropathy. J Diabetes Res. 2016;2016:5749857.

39. Iwatsuka H, Shino A, Suzuoki Z. General survey of diabetic features of yellow KK mice. Endocrinol Jpn. 1970;17(1):23-35.

40. Bazhan NM, Makarova EN. [The effect of the "yellow" mutation at the mouse agouti locus on the hormonal profile of pregnancy and lactation]. Ross Fiziol Zh Im I M Sechenova. 2009;95(11):1254-1257.
41. Herberg L, Coleman DL. Laboratory animals exhibiting obesity and diabetes syndromes. Metabolism. 1977;26(1):59-99.

42. Tong XL, Dong L, Chen L, Zhen Z. Treatment of diabetes using traditional Chinese medicine: past, present and future. Am J Chin Med. 2012; 40(5):877-886.

43. Shi X, Lu XG, Zhan LB, et al. The effects of the Chinese medicine $\mathrm{ZiBu} \mathrm{PiYin}$ recipe on the hippocampus in a rat model of diabetesassociated cognitive decline: a proteomic analysis. Diabetologia. 2011; 54(7):1888-1899.

44. Zhao HL, Sui Y, Qiao CF, et al. Sustained antidiabetic effects of a berberine-containing Chinese herbal medicine through regulation of hepatic gene expression. Diabetes. 2012;61(4):933-943.

45. Wen $X$, Zeng Y, Liu L, et al. Zhenqing recipe alleviates diabetic nephropathy in experimental type 2 diabetic rats through suppression of SREBP-1c. J Ethnopharmacol. 2012;142(1):144-150.

46. Xiao Y, Liu Y, Yu K, et al. The effect of Chinese herbal medicine on albuminuria levels in patients with diabetic nephropathy: a systematic review and meta-analysis. Evid Based Complement Alternat Med. 2013 ; 2013:937549.

47. Pirillo A, Catapano AL. Berberine, a plant alkaloid with lipid- and glucose-lowering properties: from in vitro evidence to clinical studies. Atherosclerosis. 2015;243(2):449-461.

48. Tillhon M, Guaman Ortiz LM, Lombardi P, Scovassi AI. Berberine: new perspectives for old remedies. Biochem Pharmacol. 2012;84(10): 1260-1267.

49. Yao J, Kong W, Jiang J. Learning from berberine: treating chronic diseases through multiple targets. Sci China Life Sci. 2015;58(9): 854-859.

50. Russell SB, Russell JD, Trupin KM, et al. Epigenetically altered wound healing in keloid fibroblasts. J Invest Dermatol. 2010;130(10): 2489-2496.

51. Sassoli C, Chellini F, Pini A, et al. Relaxin prevents cardiac fibroblastmyofibroblast transition via notch-1-mediated inhibition of TGF-beta/ Smad3 signaling. PLoS One. 2013;8(5):e63896.

52. Chuang PY, Menon MC, He JC. Molecular targets for treatment of kidney fibrosis. J Mol Med (Berl). 2013;91(5):549-559.

53. Kalluri R, Weinberg RA. The basics of epithelial-mesenchymal transition. J Clin Invest. 2009;119(6):1420-1428.

54. Grande MT, Sanchez-Laorden B, Lopez-Blau C, et al. Snail1-induced partial epithelial-to-mesenchymal transition drives renal fibrosis in mice and can be targeted to reverse established disease. Nat Med. 2015; 21(9):989-997.

55. Lovisa S, LeBleu VS, Tampe B, et al. Epithelial-to-mesenchymal transition induces cell cycle arrest and parenchymal damage in renal fibrosis. Nat Med. 2015;21(9):998-1009.

56. Saad S, Stanners SR, Yong R, Tang O, Pollock CA. Notch mediated epithelial to mesenchymal transformation is associated with increased expression of the Snail transcription factor. Int J Biochem Cell Biol. 2010; 42(7):1115-1122.

\section{Publish your work in this journal}

Drug Design, Development and Therapy is an international, peerreviewed open-access journal that spans the spectrum of drug design and development through to clinical applications. Clinical outcomes, patient safety, and programs for the development and effective, safe, and sustained use of medicines are the features of the journal, which

\section{Dovepress}

has also been accepted for indexing on PubMed Central. The manuscript management system is completely online and includes a very quick and fair peer-review system, which is all easy to use. Visit http://www.dovepress.com/testimonials.php to read real quotes from published authors. 